INVESTIGACIÓN

https://doi.org/10.15198/seeci.2019.49.1-18

Recibido: 02/07/2017 --- Aceptado: 17/01/2019--- Publicado: 15/07/2019

\title{
BASES CONCEPTUALES PARA EL DISEÑO DE UNA METODOLOGÍA DE DIDÁCTICA MUSICAL
}

\section{CONCEPTUAL BASES FOR THE DESIGN OF A METHODOLOGY OF MUSICAL DIDACTICS}

Ana María Botella Nicolás ${ }^{1}:$ Universitat de València. España. ana.maria.botella@uv.es

José Rafael Adell Valero²: Florida Universitària. España.

radell@florida-uni.es

\section{RESUMEN}

El objetivo principal de este artículo es diseñar una metodología adecuada para impartir música desde la escuela infantil hasta la enseñanza universitaria. Con este fin, se muestran los fundamentos teóricos que han inspirado el método. La base del mismo es la refundición de dos metodologías existentes: la cooperativa y la de proyectos. Ambas se han fusionado para su mejor aprovechamiento didáctico. Finalmente se ha esbozado su aplicación en un contexto real en dos ámbitos escolares como educación secundaria obligatoria y magisterio.

PALABRAS CLAVE: música - didáctica - aprendizaje cooperativo - aprendizaje por proyectos - metodología - enseñanza superior.

\section{ABSTRACT}

This article will attempt to demonstrate the fundamentals that have inspired a musical learning methodology aimed at any educational level. The consolidation of two existing methodologies is proposed: the first is the cooperative approach, and the second is project-based learning (PBL). Both have merged to enhance educational achievement. Finally, its application in real secondary education and teacher training degrees will be outlined.

KEY WORDS: music - teaching - cooperative learning - project learning methodology - higher education.

${ }^{1}$ Ana María Botella Nicolás: Profesora contratada doctora del Departamento de Didáctica de la Expresión musical, Plástica y Corporal de la Facultad de Magisterio de la Universitat de València. ana.maria.botella@uv.es

2José Rafael Adell Valero: Doctor en Música. Licenciado con grado en Ciencias biológicas. Profesor superior de tuba. Profesor Departamento de Educación de Florida Universitària.

radell@florida-uni.es 


\section{BASES CONCEITUAIS PARA O DESENHO DE UMA METODOLOGIA DE DIDÁTICA MUSICAL}

\section{RESUME}

O objetivo principal deste artigo é desenhar uma metodologia adequada para ensinar música da escola infantil até a universidade. Com este fim, mostramos os ensinamentos teóricos que inspiraram o método. A base do mesmo é a refundição de duas metodologias existentes: a cooperativa e a de projetos. Ambas se fusionaram para seu melhor aproveitamento didático. Finalmente foi desenhado sua aplicação em um contexto real em dois âmbitos escolares como educação segundaria obrigatória e magistério.

PALAVRAS CHAVE: música - didática - aprendizagem por projetos - metodologia - ensinamento superior.

\section{Cómo citar el artículo:}

Botella Nicolás, A. M., y Adell Valero, J. R. (2019). Bases conceptuales para el diseño de una metodología de didáctica musical. [Conceptual bases for the design of a methodology of musical didactics]. Revista de Comunicación de la SEECI, 49, 1-18. doi: http://doi.org/10.15198/seeci.2019.49.1-18

Recuperado de http://www.seeci.net/revista/index.php/seeci/article/view/487

\section{INTRODUCCIÓN}

Existen momentos importantes en la vida de las personas, en los que se han de tomar decisiones que afecten a su futuro y que, sin duda, encaminarán sus esfuerzos hacia objetivos y actuaciones concretas. Dedicarse a la enseñanza es uno de ellos porque, indudablemente, el compromiso que se produce, afectará a su destino. No cabe duda que es una decisión importante. Y más si se trata de la enseñanza de la Música.

En cualquier materia ha habido una actualización sustancial desde mediados del siglo XX, pero en este caso, los estudios musicales han dado un giro extraordinario, debido, sobre todo, a que al situarse en el currículum escolar desde la Educación Infantil hasta la secundaria obligatoria inclusive, no era posible continuar impartiendo los contenidos y la metodología, de la misma manera que se venía haciendo hasta ahora. Hay que pensar en construir otro sistema distinto al que estaba en vigor, sobre todo en los primeros niveles de aprendizaje. Por ello, el principal reto se produce, en las Escuelas de Magisterio donde hay que preparar al alumnado para que contemple otras alternativas que, además de motivar el interés de sus alumnos y la adquisición de conocimientos musicales, colabore en el desarrollo integral de las personas.

\section{APORTACIONES TEÓRICAS RELEVANTES}

Descubrir que esta nueva aplicación de la teoría musical es factible, adecuada e incluso necesaria es fundamental. Se había producido una transformación del 
concepto de la enseñanza de la Música desde mediados del siglo XX muy importante, pero a nuestro país llegaba con mucho retraso y en condiciones poco favorables para su experimentación. La causa de esta transformación había sido la confluencia o relación entre pedagogos y compositores quienes consiguieron dar forma a nuevos enfoques y sentar las bases de una pedagogía actualizada, moderna y acorde con los tiempos. Los movimientos más importantes habían partido de Europa y América, y diversos autores estaban comprometidos en esta importante transformación. A veces, aparecían casos aislados, pero, en conjunto, parecía que una corriente de energía les conectaba a todos, puesto que con pequeñas variantes coincidían en lo fundamental. Uno de los referentes importantes fue Murray Schafer que desde 1965 trabajaba intentando restablecer la unidad musical, y recuperar la música y el increíble mundo de los sonidos para las jóvenes generaciones.

El cambio que se estaba produciendo respondía a la ampliación de la función de la educación musical en todos los niveles educativos. Hasta ahora se había considerado que la Música era un lenguaje capaz de desarrollar la sensibilidad y de construir formas estructuradas de pensamiento, acordes con las demás materias. Esos fundamentos a pesar de ser precisos, no obstante, resultan insuficientes cuando hay que extenderlos a todas las etapas educativas.

Schafer (2008, p. 32) amplió este campo de acción al cuestionarse: "¿Por qué enseñar música? ¿Qué debería enseñarse? ¿Cómo debería enseñarse? ¿Quién debería enseñarla?". Escribió varios libros en los que iba respondiendo a estas preguntas a través de experiencias activas motivadas por la búsqueda de nuevas soluciones metodológicas innovadoras: "iporqué" y "para qué"? He aquí dos preguntas fundamentales para comenzar un cambio. En palabras de Delalande: "Me parece que los músicos, ya hagan, ya escuchen, tienen en común esas tres grandes capacidades: ser sensibles a los sonidos, encontrar en ellos una significación y gozar de su organización"(Delalande, 1995, p. 11).

En esencia, la innovación pedagógica que se perseguía consistía en cambiar fundamentalmente dos principios: el concepto y la metodología. De esta manera, se pretendía potenciar:

- La investigación sobre el sonido, natural o artificial, lo cual le daba otra dimensión sonora al hecho musical.

- Intensificar la creatividad en la enseñanza, tanto musical como en relación con otras formas artísticas, cosa que permite enfocar la asignatura con otro talante más innovador, espontáneo y sensible.

- Aprovechar la inclusión de otras formas procedimentales para desarrollar características sonoras a través de materiales que permitan la introducción de nuevos aspectos sonoros expresivos y experimentales. Es decir, buscar el sonido en todos los elementos que puedan producirlo para agregarlo como experiencia sensible a otras semejantes.

- Examinar la naturaleza de los sonidos del entorno y sensibilizarse para ser conscientes de los problemas de la polución sonora y de los "ruidos" que pueden ser aprovechables para efectos expresivos. En consecuencia, 
proponer una comprensión fundamental: es necesaria una regeneración sonora ambiental.

No obstante, Schafer (2008), aún va más lejos en sus investigaciones ya que determina acabar con las dicotomías existentes entre música escolar y música viva, música vieja o de museo y música nueva, música culta y música popular... y establecer un nuevo acercamiento al fenómeno sonoro. De esta manera, considera que el sonido es un elemento fundamental en su propuesta experimental y lo antepone a lo que ha venido tradicionalmente en denominarse Música, sobre todo si se habla de Música clásica, sinfónica o culta. En realidad, fue la persona necesaria para empujar una transformación del concepto musical, por lo que dedicó sus energías a divulgar sus experiencias en universidades europeas, en las que expuso sus planteamientos revolucionarios.

Otro de sus principales objetivos consiste en profundizar en lo que denomina Paisaje Sonoro (soundscape) y que consiste en descubrir y utilizar aquellos sonidos o ruidos que produce el medioambiente y que forman, todos juntos, una composición universal. Al hacerlo distingue los sonidos principales producidos por un medio natural o artificial, las señales sonoras que se sitúan en primer plano, o las marcas sonoras, cuyo sonido único le hace representar a un lugar concreto. Desde estas premisas se pregunta "¿No podría ser enseñada la música como una disciplina que simultáneamente libera energías creadoras y educa la mente para la percepción y el análisis de sus propias creaciones?" (Schafer, 2008, p. 22).

Se esbozan pues, dos cuestiones fundamentales: por un lado, liberar energías creadoras y por otro, educar, distinguiendo claramente las funciones del ruido y del sonido para aprovechar ambas funcional y creativamente.

Sin embargo, algo que resulta tan evidente a veces, fue mal interpretado o seguido de manera poco efectiva ya que, debido a la novedad de los planteamientos en el panorama musical educativo, habían surgido docentes que, por su improvisación y falta de sistematización metodológica, hacían tambalearse el edificio construido con tanta eficacia y claridad por los autores mencionados.

Es imposible, acotar todos sus pensamientos y menos en este espacio tan reducido, pero deberíamos considerar y reflexionar seriamente sobre sus propuestas innovadoras, buscando la manera idónea y personal de llevarlas a cabo. Por supuesto, es difícil, pero hay que ajustarse al cambio de los tiempos y seguir los nuevos descubrimientos que aportan nuevas luces al quehacer pedagógico. Esto confirma la necesidad de continua renovación a la que deben adaptarse los profesores de cualquier ámbito, disciplina o nivel.

Además, sería conveniente recordar que, en el caso de los profesores universitarios, la formación se debe dar a tres niveles: el estudio, la docencia y la investigación y que nunca acabaremos en un punto determinado del camino de la formación puesto que éste no es una visión aparente de la realidad como las paralelas que se unen en el infinito. 


\section{RECURSOS INNOVADORES EN LA DOCENCIA: EL MÉTODO}

No es posible evocar nuestros primeros recuerdos sonoros. A veces podemos remontarnos hasta los 3 años cuando algo nos sorprendió extraordinariamente. Pero si hacemos un esfuerzo y nos situamos en edades tempranas, comprenderemos que ante la inmaculada blancura de una mente que empieza a despertar, cualquier experiencia puede ser, no sólo gratificante, sino también emocionante. Y esa emoción es la que va a configurar y ayudar a construir nuestra personalidad. No obstante, es necesario que la enseñanza de la Música no se convierta en un sistema aleatorio en el que falte la articulación de experiencias que sean coherentes entre sí. Una cosa es facilitar el estudio de algo tan específico y abstracto como el lenguaje musical y otro dejar a la arbitrariedad y a la improvisación la realización de ejercicios que estén situados fuera del currículum y que sean una mera distracción para los alumnos.

Ha de haber, pues, un hilo conductor que el profesor debe tener siempre presente para que la enseñanza-aprendizaje sea productiva. $Y$ para transmitir los contenidos se necesita un método, abierto y flexible, que permita el intercambio, la cooperación y la asimilación del conocimiento, en grupo e individualmente. Porque, además de los contenidos que se impartan, el carácter de la asignatura permite actuar sobre los alumnos formando grupos cooperativos a fin de que muchas cualidades que son factibles de desarrollar: socialización, integración en un trabajo común, autoestima, flexibilidad... pasen a formar parte de los conocimientos que se adquieran y que son fundamentales en una sociedad democrática.

En el caso de la Música, escuchar, identificar y producir sonidos, tocar, apreciar cualidades, favorecer el sentido espacial, el movimiento, la dirección... es fundamental. La sensibilidad hacia el sonido, y la diferenciación de sus características básicas: altura, duración, intensidad y timbre, pueden enriquecer la identificación y descripción de los sonidos. También es importante hacer hincapié en el reconocimiento y descripción de algunos elementos y formas de organización y estructuración musical, como la melodía, la textura, la repetición, la imitación y las variaciones de una obra. Como se puede ver, hay muchos elementos que trabajar y esto debe hacerse en profundidad, pero tratando de que el alumnado lo reciba como una experiencia gratificante y enriquecedora.

¿Cómo se podía llevar a la práctica la teoría elegida? Hubo que plantearse pues, cuál sería el método más idóneo para desarrollar el campo de competencias que se deberían trabajar y el mayor provecho que se podía obtener a través de la Música. Es evidente que los contenidos conceptuales y la metodología musical, debían estar estrechamente unificados. De manera que, después de cotejar varias opciones, se eligió el Método Cooperativo por Proyectos, que se adaptaba con garantías de éxito a las aspiraciones que se pretendían alcanzar.

Como se puede comprender tomar la decisión de basarnos en el Método Cooperativo por Proyectos se hizo después de muchas investigaciones, dudas y reflexiones. También resultó evidente que los dos elementos separados (Método 
Cooperativo y Método por Proyectos), eran insuficientes para conseguir los objetivos propuestos, de manera que no hubo ninguna duda en fusionarlos para obtener mejores resultados educativos.

Tal y como afirman Botella y Adell (2016) no existe la denominación de este método como tal. En realidad, se encuentran separados el Aprendizaje Cooperativo y el de Proyectos, aunque sea posible su refundición. En este caso concreto, ambos métodos se han combinado complementándose puesto que juntos, pueden ofrecer un mayor número de posibilidades educativas, al no ser excluyentes.

¿Qué pretende aunar el método elegido? Por un lado, el trabajo en grupos cooperativos $y$, por otro, que la experiencia se desarrolle como un proyecto previo al cual cada alumno aportará sus conocimientos. Además, cualquier circunstancia que ocurra fuera aula, podrá ser aprovechada por todos cuando se ponga en común. De esta manera no sólo se trabajarán aspectos que se desarrollarán en clase sino que, además, el alumno tendrá que esforzarse en aportar datos de interés respecto al entorno sonoro de su casa, su calle, su barrio, la naturaleza que pueda haber a su alrededor, televisión, etc... es decir, analizando e incorporando en clase aquellos elementos del "paisaje sonoro" con los que convive. En ambos casos se trata de trabajar de manera abierta fomentando la curiosidad, la experimentación y el desarrollo de estrategias que llevan a la obtención de resultados óptimos. Piaget comenta:

El principal objetivo de la educación es crear hombres que sean capaces de hacer cosas nuevas, no simplemente repetir lo que otras generaciones han hecho, hombres que sean creativos, inventivos, descubridores. El segundo objetivo es formar mentes que puedan ser críticas; que puedan verificar, y no aceptar todo lo que se les presenta (...) Necesitamos alumnos que sean activos, que aprendan temprano a investigar por sí mismos, en parte a través de su propia actividad espontánea, y en parte a través del material que les presentamos. (Piaget 1964, citado en Siegel y Brainerd, 1978, p. 32).

De esta manera, si conjugamos los dos métodos, se aprecia un efecto sinérgico basado en fomentar el trabajo entre iguales, la negociación permanente, la socialización de los alumnos, la necesidad de una organización sujeta a los objetivos y recursos. No debe faltar la curiosidad, el descubrimiento, la planificación del trabajo, la atención a la diversidad, así como la posibilidad de integrar a alumnos con necesidades educativas especiales. Falta por añadir que el profesor, en este caso, juega un rol de guía en el proceso de metacognición.

\subsection{Características más importantes del método cooperativo}

El Aprendizaje Cooperativo supone el empleo didáctico de grupos reducidos donde los alumnos trabajan juntos para maximizar su propio trabajo y el de los demás. Por otra parte, implica la realización de un esfuerzo que influye sobre muchos resultados diferentes al mismo tiempo. De ahí su gran capacidad para generar respuestas divergentes, creativas y socializadoras, que enriquecen el acto educativo.

Revista de Comunicación de la SEECI. 15 julio, 2019 / 15 noviembre, 2019, nº 49, 1-18 
Torrego y Negro (2012, p. 15) señalan que la cooperación: "No es sólo una alternativa metodológica y potencialmente eficaz para enseñar, sino una estructura didáctica con capacidad para articular los procedimientos, las actitudes y los valores propios de una sociedad democrática que quiere reconocer y respetar la diversidad humana". En esta situación, los individuos procuran obtener resultados que sean beneficiosos para ellos mismos y para todos los demás miembros del grupo.

Por tanto, es más que una agrupación de personas puesto que hay que integrar individuos aislados que deben compartir espacios físicos y objetivos genéricos. Además, sus componentes deben adoptar roles funcionales, establecer una comunicación fructífera y aunar esfuerzos para obtener resultados óptimos. "La implementación en las escuelas de las técnicas de aprendizaje cooperativo, son altamente eficaces para mejorar (...) la motivación intrínseca, la autoestima y el funcionamiento de las capacidades intelectuales, aumentando particularmente las capacidades críticas y la calidad del procesamiento cognitivo de la información" (Ovejero, 1993, p. 387).

Podríamos decir que un antecedente fundamental en este tipo de aprendizaje lo constituye la teoría genética de Piaget (1896-1980). Él marca un antes y un después en la educación al introducir en la metodología la construcción, o secuencia ordenada de estructuras intelectuales que, de alguna forma, regulan los intercambios funcionales o comportamentales de las personas con su medio.

En EEUU a partir del siglo XIX se empieza a difundir el aprendizaje cooperativo, pero no fue hasta el siglo XX con el filósofo John Dewey (1859-1952), cuando se elaboró un proyecto metodológico de instrucción, en el que se promovió el uso de los grupos de aprendizaje colaborativo. Así se cambió la concepción del sujeto individual para transformarse en órgano de la sociedad. Este nuevo planteamiento requiere que el individuo sea preparado con la finalidad de aportar. Dewey revoluciona la educación e introduce la experiencia como parte de ella. Lo social ocupa un lugar relevante, sentando las bases para la creación de una escuela activa, de la misma forma que recalca la importancia de la cooperación frente al individualismo, la creatividad frente a la pasividad y el trabajo manual frente a las asignaturas. Otro aspecto importante lo asigna a los libros de texto, a los que confiere solo la función de consulta. Estas ideas de Dewey lo sitúan como el verdadero creador de la llamada Escuela Activa, que serviría de estímulo a la creación del Plan Dalton, el Método de Cousinet y su famoso Método de Proyectos.

La historia de los antecedentes del trabajo grupal en centros de enseñanza revela que uno de los métodos que con mayor frecuencia se aplicaban en la práctica pedagógica, a través de todo el siglo XX fue el Método de Aprendizaje Colaborativo. Éste es por lógica, la continuidad de la concepción del aprendizaje activo que arraigó en los Estados Unidos en toda esta etapa; pero redimensionado a partir de los resultados de los experimentos clásicos de la psicología social acerca de las estructuras grupales competitivas, colaborativas y cooperativas. 
Howard Gardner (1943-2011) también construye una teoría, la de las inteligencias múltiples, puesto que según él, la inteligencia no es una entidad unitaria, sino que se compone de una suma de diversas inteligencias (ocho en total) con características y evolución propia.

En los años setenta la investigación y práctica de los métodos cooperativos cobran gran relevancia. Continúa evolucionando la teoría y aplicación de las técnicas cooperativas en el ámbito escolar sobre todo en Estados Unidos y Canadá. Los aportes del "Método de Aprendizaje Colaborativo", han sido retomados a partir de la década de los años 90 en diferentes niveles de enseñanza y se destacan como partidarios de sus premisas: E. Cohen y D. M. Evans en EE. UU; T. Ryoko y Y. Kobayashi en Japón; A. Álvarez en España y R. Ferreiro Gravié en Cuba.

Otras investigaciones llevadas a cabo en el aprendizaje de proyecto cooperativo de carácter internacional cuenta entre sus mayores expertos, según Torrego y Negro: "En primer lugar a los hermanos Roger. $T$ y David W. Johnson (Jhonson, Johnson y Holubec, 1997; Johnson y Johnson, 2008), en segundo lugar al profesor Spencer Kagan (Kagan, 2001) y finalmente al profesor Robert Slavin (Slavin, 1995)" (Torrego y Negro, 2012, p. 26). Todos ellos coinciden en las condiciones básicas para aprender cooperando y andamiar los conocimientos, con objeto de que el aprendizaje se vaya construyendo sobre unas bases sólidas y significativas. En cuanto a España conviene destacar "los trabajos pioneros de Ovejero (1990) o de Echeita y Martín (1990), Echeita (1995), o los más recientes de Díaz Aguado (2003), David Durán (Durán y Vidal, 2004), Pere Pujolàs (2004; 2009) o Moruno, Sanchez y Zariquiey (2011)"(Torrego y Negro, 2012, p. 26).

Las tendencias actuales del aprendizaje cooperativo son las siguientes: El Learning together de D.W. Johnson y R. T. Johnson, el Student Team Learning de Slavin, el Group Investigation de Sharan y Sharan, la Structural Approach de Kagan y Kagan, la Complex Instruction de Cohen y la perspectiva del Collaborative Approach.

El aprendizaje cooperativo puede entenderse como un bloque de técnicas basadas en los principios teóricos interaccionistas. Es decir, dado que el ser humano y sus elementos constitutivos se desarrollan a lo largo de la interacción social "la solución a los problemas intelectuales, de personalidad o motivacionales que están en la base del fracaso escolar pasa ineludiblemente por una sustancial mejora de la interacción social de quienes fracasan en la escuela"(Ovejero, 1993, p. 387).

En cuanto a los objetivos del aprendizaje cooperativo, diversos autores han definido su naturaleza: A continuación, se presentan algunos ejemplos, así como las condiciones que se suponen básicas en este tipo de aprendizaje.

- Tratándose de equipos de aprendizaje cooperativos el primer objetivo es obvio: progresar todos en el aprendizaje; saber, al final de cada unidad didáctica y al final de un curso académico determinado, más de lo que sabían al iniciarlo, cada uno según sus capacidades; no se trata de que todos aprendan lo mismo, sino de que cada uno progrese todo lo que pueda en su aprendizaje. 
- Y tratándose de equipos de aprendizaje cooperativos, el segundo objetivo es igualmente claro: "ayudarse unos a otros, cooperar, para progresar en el aprendizaje"(Pujolàs, 2012, p. 92).

En la actualidad, las cinco condiciones básicas que caracterizan y sustentan el aprendizaje cooperativo las podemos considerar como: Interdependencia positiva entre los participantes, responsabilidad personal y rendimiento individual, interacción promotora, habilidades sociales y evaluación periódica (Echeita, 2012).

Otros autores (Borrás y Gómez, 2010), presentan estas cinco condiciones básicas así: Interdependencia positiva, responsabilidad individual y grupal, interacción estimuladora, aprender algunas prácticas interpersonales y evaluación grupal.

Como se puede observar, aunque los autores sean distintos, las propuestas que plantean son bastante similares. En ambos casos se destaca, además de la interdependencia positiva y de la interacción positiva o promotora, la responsabilidad que cada miembro del grupo debe asumir para que se den resultados óptimos. Si no hay una práctica que se remonte a otras experiencias educativas similares, la tarea puede ser bastante compleja y requerirá una mayor duración temporal en el caso de cada actividad. Por ello, es necesario que se produzca una adaptación hacia el comportamiento socio-grupal en el que se irá aprendiendo mediante la aplicación de algunas prácticas interpersonales. En cualquier caso, la evaluación será un factor importante que ayudará a los alumnos y a los profesores a ir asumiendo parcialmente al principio y en conjunto después, los resultados que se vayan produciendo en el trabajo realizado de forma cooperativa.

Como se deduce de lo anteriormente expresado, no es posible la improvisación, aunque algunos factores aleatorios pueden tener su importancia. Es necesario, pues, partir de una "estructura", es decir de un conjunto de actividades que buscan una finalidad y están socialmente organizadas. Las actividades y la finalidad están relacionadas con la implementación y el sostén de la interdependencia positiva. Hay que pensar en la heterogeneidad de alumnos en las aulas y en su inclusión total en el aprendizaje. Cada alumno aprende de un modo distinto.

En cuanto a las estrategias que ayudarán a la implementación del proyecto cooperativo, se fundamentarán en aquellas que mantengan una forma de interdependencia entre los alumnos. Por ello irán destinadas a:

- Compartir objetivos comunes personalmente aceptados y valorados.

- Compartir medios y recursos necesarios para una tarea compleja.

- Estructurar las tareas de aprendizaje y de evaluación de modo interdependiente. Para ello, (...) se han creado múltiples estructuras, simples y complejas, que permiten, (...) algo así como un "andamiar", garantizar interacciones positivamente facilitadoras del aprendizaje y de la concentración en la tarea.

- Reforzar el reconocimiento, el esfuerzo y las recompensas grupales.

- Asumir símbolos y señas de identidad grupal: nombres de equipo, logos, lemas, etc. 
- Celebrar el éxito de cada uno como el del colectivo, y de éste como algo personal (Echeita, 2012, p. 28).

Las emociones y las actitudes que se vinculan a esta condición se podrían resumir como "nos necesitamos unos a otros y todos podemos aportar" (Echeita, 2012, p. 28). El trabajo en equipo puede considerarse como un contenido a enseñar. Según Pujolàs compilado en Torrego y Negro, deben destacarse también como estrategias: La cohesión del grupo, el progreso de todos en el aprendizaje, autorregularse como equipo, autoevaluación continua, definición de conflictos y empleo de las TIC como registro del aprendizaje cooperativo.

La dinámica grupal presenta las siguientes características:

El trabajo se desarrollará en pequeños grupos en los que los alumnos aprenden unos de otros, mediante la interacción entre iguales. Los miembros del equipo deben ser conscientes de que su rendimiento depende del esfuerzo de todos. El objetivo grupal de maximizar el aprendizaje de todos motiva a los miembros a esforzarse y a obtener resultados que superen la capacidad individual de cada uno de ellos. Si uno fracasa, todos fracasan. Cada miembro del grupo asume responsabilidad y hace responsable al resto del grupo para conseguir objetivos comunes. Se trabaja conjuntamente con la finalidad de producir resultados de conjunto. Se da un apoyo recíproco, se ofrecen ayudas, explicaciones, etc. Se utilizan ciertas formas de relación interpersonal, como el reparto de tareas y responsabilidades, para coordinar el trabajo y conseguir los objetivos. (Johnson, Johnson, y Holubec, 1999, citado por Borrás y Gómez, 2010, pp. 9-11).

Como se deduce de estas premisas, el aprendizaje cooperativo es más que una alternativa metodológica ya que resulta potencialmente eficaz en la enseñanza y, además, crea un espacio con capacidad para articular las actitudes y los valores propios de una sociedad democrática en la que se reconoce y respeta la diversidad humana. El grupo cooperativo analiza la eficacia con la que consigue los objetivos y el proceso de trabajo conjunto para garantizar el progreso continuo del aprendizaje (Johnson, Johnson y Holubec, 1994). La idiosincrasia del trabajo cooperativo hace que se desarrollen una serie de competencias básicas sólo por el hecho de utilizar esta metodología:

- Comunicación lingüística: se requiere una expresión oral y escrita que vehicule el aprendizaje que se pretende realizar. Uso de una terminología adecuada y un vocabulario básico que se debe manejar con fluidez.

- Tratamiento de la información: es fundamental hacer un buen uso de las fuentes de información así como un buen filtrado de las mismas. En esta competencia es fundamental conocer y hacer un uso adecuado de las TIC.

- Social y ciudadana: el hecho de trabajar en grupo supone una continua interacción con los miembros del mismo. Hay un continuo intercambio de opiniones, criterios y es imprescindible llegar a acuerdos donde todas las partes se sientan satisfechas. 
- Cultural y artística: muy a menudo este tipo de trabajos terminan en un producto que requiere de una habilidad artística que lo desarrolle de forma creativa y conveniente.

- Aprender a aprender: la autonomía de los grupos es fundamental, siendo el profesor un acompañante o guía. Esto supone que los alumnos se ven inmersos en un proceso en el cual ellos son en gran medida los responsables de su aprendizaje.

- Autonomía e iniciativa personal: Aunque parezca un contrasentido, es fundamental la iniciativa personal y el empuje de cada uno de los miembros del grupo para conseguir su avance. Se trata de aprovechar las potencialidades de cada uno de los miembros del mismo para rentabilizar esfuerzos consiguiendo resultados óptimos.

El resto de competencias básicas se trabajan en mayor o menor medida en función del contenido del aprendizaje en cuestión.

Otro aspecto a destacar en esta forma metodológica es la evaluación. La evaluación grupal sin dejar de señalar que se debe partir de un estudio de las características del alumnado antes de establecer cualquier criterio de valoración. Por tanto, deberán considerarse a nivel general:

- Qué conocimientos previos se encuentran en el punto de partida del tema elegido, tanto a nivel teórico como práctico (motivación intereses, etc...)

- Cómo se encuentran los alumnos respecto a su grado de cooperación en el estadio evolutivo en que se encuentren.

- Características de las diversas personalidades, introversión, extraversión, capacidad de liderar grupos, etc.

- Cuáles son las capacidades específicas que pueden adaptarse a sus necesidades educativas.

También es muy importante en la evaluación grupal hacer un seguimiento de cualquier incidencia significativa con el apoyo de las TIC que nos darán una visión completa de los avances, dudas, retrocesos, intervenciones, liderazgos... que se produzcan a medida que el trabajo progrese.

\subsection{Características más importantes del método de proyectos}

Según Katz, citado en Clark (2006) un proyecto consta de una investigación profunda, realizada por el alumnado, de un tema digno de su tiempo, atención y energía. Esta definición un tanto simplificada deberá ampliarse para su mejor comprensión. Tal y como indica Clark (2006) un proyecto incluye tres fases:

- Los alumnos junto a su maestro eligen y discuten un tema a explorar.

- El paso siguiente consiste en que los alumnos realizan investigaciones directas y después organizan y disponen sus hallazgos.

- El proyecto culmina con una serie de preguntas y respuestas así como de la puesta en común de sus investigaciones. 
En parte, esta división de secuencias se debe a John Dewey (1859-1952), el cual afirmaba que la educación consta de la organización y de la reconstrucción de la experiencia. De esta manera, para él, la adquisición del conocimiento se hace como un redescubrimiento personal. Hacia finales del XIX se amplió a la rama de ingeniería y se expandió por Europa y América. En 1879 C. M. Woodward fundó la primera Manual Training School en San Luis con clara influencia rusa en la que los procesos derivaban desde la instrucción a la construcción.

John Dewey se interesa por el método haciendo hincapié en la creatividad. Pero la nueva demanda de ampliación hacia el conocimiento de una nueva psicología de la educación no se produjo hasta que Rufus W. Stimson del Massachusetts Board of Education no realizó su proyecto Home Project Plan adaptado a la agricultura en 1910.

Sin embargo, el término proyecto no se aplicó de un modo más general hasta que William H. Kilpatrick publicó su ensayo The Project Method en 1918. Para él los proyectos quedaban definidos por cuatro fases: la motivación, el planteamiento, la ejecución y el juicio crítico. Dewey, maestro de Kilpatrick, criticó el proyecto de su alumno y el método cayó en desuso. Aún sí, tras muchas vicisitudes, a principios del siglo XX el método volvió a cobrar relevancia y fue considerado en países como Canadá, Argentina, Reino Unido, Alemania, India y Australia. Curiosamente el centro de discusión se ubicó en Rusia donde, desde los años veinte, se consideró como una alternativa a la enseñanza capitalista. En los años treinta la metodología por proyectos era considerada como el único medio realmente marxista y democrático de enseñar. Se mantuvo durante una década hasta después de la segunda guerra mundial. Durante los años sesenta el Método de Proyectos se expandió por toda Europa actualizando el sistema que anteriormente habían ideado Kilpatrick y Dewey y aún permanece activo en Alemania. Por supuesto, estas apreciaciones se dedicaron al estudio general de cada materia y no fueron adaptadas específicamente al mundo musical.

En cuanto a sus objetivos y Según Clark (2006) a la hora de emprender un proyecto, además de las tres fases del mismo, necesitan considerarse tres componentes: el contenido, los procesos y los productos.

Los proyectos de trabajo suponen una manera de entender el sentido de la escolaridad basado en la enseñanza para la comprensión, lo que implica que los alumnos participen en un proceso de investigación, que tiene sentido para ellos (no porque sea fácil o les guste) y en el que utilizan diferentes estrategias de estudio. Pueden participar en el proceso de planificación del propio aprendizaje, y les ayuda a ser flexibles, reconocer al otro y comprender su propio entorno personal y cultural. Esta actitud favorece la interpretación de la realidad, orientada hacia el establecimiento de relaciones entre la vida de los alumnos y profesores y el conocimiento que las disciplinas y otros saberes no disciplinares, van elaborando.

Todo ello para favorecer el desarrollo de estrategias de indagación, interpretación y presentación del proceso seguido al estudiar un tema o un problema, que por su 
complejidad favorece el mejor conocimiento de los alumnos y los docentes de sí mismo y del mundo en el que viven ${ }^{3}$.

En cuanto a las estrategias, el método se concreta como:

- Un conjunto de atractivas experiencias de aprendizaje que involucran a los alumnos en proyectos complejos y del mundo real a través de los cuales desarrollan y aplican habilidades y conocimientos.

- Una estrategia que reconoce que el aprendizaje significativo lleva a los estudiantes a un proceso inherente de aprendizaje, a una capacidad de hacer trabajo relevante y a una necesidad de ser tomados seriamente.

- Un proceso en el cual los resultados del programa de estudios pueden ser identificados fácilmente, pero en el cual los resultados del proceso de aprendizaje de los estudiantes no son predeterminados o completamente predecibles.

- Este aprendizaje requiere el manejo, por parte de los estudiantes, de muchas fuentes de información y disciplinas que son necesarias para resolver problemas o contestar preguntas que sean realmente relevantes.

- El método de proyectos es una estrategia de aprendizaje que se enfoca a los conceptos centrales y principios de una disciplina, involucra a los estudiantes en soluciones de problemas y otras tareas significativas, les permite trabajar de manera autónoma para construir su propio aprendizaje y culmina en resultados reales generados por ellos mismos.

- El trabajar con proyectos puede cambiar las relaciones entre los maestros y los estudiantes.

- Puede también reducir la competencia entre los alumnos y permitir a los estudiantes colaborar, más que trabajar unos contra otros. Además, los proyectos pueden cambiar el enfoque del aprendizaje, lo pueden llevar de la simple memorización de hecho a la exploración de ideas ${ }^{4}$.

En el proceso de aprendizaje de los alumnos se dan las siguientes etapas:

Desarrollar sus propias preguntas acerca del tema de investigación, hacer predicciones acerca de posibles respuestas, idear maneras de poner a prueba sus hipótesis, lograr un acuerdo con el maestro acerca de varias maneras de representar sus hallazgos, y tomar tiempo para resolver sus propios problemas por ensayo y error. (Clark, A. 2006, p. 1).

Las fases que desarrolla Clark se consideran el ideal dentro de las etapas de aprendizaje de los alumnos, pero no siempre coinciden con la realidad que se da en el aula:

El planteamiento que inspira los proyectos de trabajo está vinculado a la perspectiva del conocimiento globalizado y relacional (...) Esta modalidad de

\footnotetext{
${ }^{3}$ Extraído el 15-4-17 de http://www.eumed.net/rev/ced/26/arsv2.htm

${ }^{4}$ Extraído el 15-4-17 de http://elmetodode.blogspot.com.es/2012/12/que-es-el-metodo-deproyectos.html
} 
articulación de los conocimientos escolares es una forma de organizar la actividad de enseñanza y aprendizaje que implica considerar que dichos conocimientos no se ordenan para su comprensión de una forma rígida, ni en función de unas referencias disciplinares preestablecidas o de una homogeneización del alumnado. La función del proyecto es favorecer la creación de estrategias de organización de los conocimientos escolares en relación con: el tratamiento de la información y la relación entre los diferentes contenidos en torno a problemas o hipótesis que faciliten al alumnado la construcción de sus conocimientos la transformación de la información procedente de los diferentes saberes disciplinares en conocimiento propio. (Hernández y Ventura, 1998, p. 47).

De la misma manera que en el aprendizaje cooperativo, en este caso también es fundamental descubrir cuál va a ser la estructura que guiará el contenido del trabajo constituyendo unas sucesiones lógicas y secuenciales y en todo caso actuará como un nexo que lo relacione con otras materias. En cualquier disciplina se considera que el tema elegido se puede rastrear en otros medios de comunicación externos al ámbito escolar, ampliando así la visión de los alumnos y su conexión con los medios informativos que se pueden encontrar en la televisión, el cine, o en cualquier otra manifestación del entorno habitual. Se trata de darle sentido al aprendizaje significativo tomando como principio básico su articulación configurada a partir de la previsión abierta. Por tanto, se evidencia la necesidad de dar un alto sentido a la funcionalidad de lo que hay que aprender y también el valor de la memorización para afianzar lo aprendido mientras se vislumbran otras relaciones en el aprendizaje.

La información necesaria para construir los proyectos no está predeterminada de antemano, ni depende del enseñante o de un libro de texto, sino que está en función de lo que ya sabe cada alumno sobre un tema y de la información con la que se pueda relacionar dentro y fuera de la escuela. Por el contrario, hay que abordar la información presentada en clase de forma que el alumnado llegue a ordenarla, valorarla e inferir de ella nuevos sentidos, significados o referencias. De manera que, en este sistema, es la clase quien elige el tema, siempre atendiendo a las propuestas del profesor, puesto que éste debe exponer las razones que determinan la elección del trabajo que se va a realizar y aportar las situaciones que deben presidirlo. Así pues, se especificará el hilo conductor de la actividad, así como la elección de los materiales con los que se van a trabajar elementos como estructura, ritmo...etc. Dichos materiales se buscarán y se elegirán entre todos, procurando que con ellos se consigan unos objetivos determinados. También será importante considerar si la relación entre el tema y los materiales serán idóneos para conseguir que aporten algo a cada alumno y al sistema de proyectos. Y, finalmente, el resultado deberá ser una experiencia original, planteada en función de una serie de conceptos que queremos desarrollar en este caso. A este respecto el profesor deberá ser paciente e insistir en la reestructuración, replanteamiento o la modificación de los elementos del proyecto, puesto que no todos los alumnos tienen la misma capacidad expresiva, rítmica o auditiva para seguir a los más dotados musicalmente. En consecuencia, es imprescindible que intervengan todos los alumnos y que el ambiente esté 
Botella Nicolás, A. M., y Adell Valero, J. R. Bases conceptuales para el diseño de una metodología de didáctica musical

impregnado de una emoción que haga a los participantes desear comunicarse y realizar un esfuerzo común.

Los procedimientos ayudan a los alumnos a incorporar nuevas estrategias de aprendizaje y al estar comprendidas en el proceso de construcción del proyecto pueden ser utilizadas en el caso concreto que se desarrolla o en otras ocasiones:

Para Nisbet y Shucksmith (1987) las estrategias de aprendizaje son 'estructuraciones de funciones y recursos cognitivos, afectivos o psicomotores que el sujeto lleva a cabo en los procesos de cumplimiento de objetivos de aprendizaje'. La forma en que operan las estrategias es mediante la plasmación de 'configuraciones de funciones y recursos, generadores de esquemas de acción para un enfrentamiento más eficaz y económico de situaciones globales y específicas de aprendizaje, para la incorporación selectiva de nuevos datos y su organización. (Hernández y Ventura, 1998, pp. 17-18).

La idiosincrasia del trabajo por proyectos hace que se desarrollen las mismas competencias básicas y de modo similar a lo expuesto en el aprendizaje cooperativo. En cuanto a la evaluación se justifica por el análisis del proceso seguido a lo largo de toda la secuencia y de las interrelaciones creadas en el aprendizaje. Por tanto, se han de anticipar decisiones, establecer relaciones e inferir nuevas cuestiones. "El Buck Institute for Education señala que en el Método de Proyectos son importantes dos tipos de evaluación: Del aprendizaje del alumno. Del proyecto"5.

En el primer caso habrá que prestar atención a los resultados así como a la autoevaluación que realicen los alumnos.

En el segundo caso y puesto que los estudiantes son, generalmente, los mejores críticos del proyecto se atenderá a su opinión. Por ello, inmediatamente después de realizado el proyecto es importante que se reflexione acerca de los éxitos o los fracasos del mismo. La autoevaluación deberá actuar como un elemento más del proceso de aprendizaje y será muy útil anotar qué cosas funcionaron bien y cuáles no, a fin de reorganizar nuevas estrategias y acciones para posteriores actividades.

\section{CONCLUSIONES Y APLICACIÓN PRÁCTICA}

En resumen, ambas metodologías tienen elementos en común que permiten, como ya se ha indicado, su fusión y puesta en marcha conjunta. La flexibilidad del Método de Proyectos combinada con la ayuda entre iguales del Método Cooperativo son factores que actúan sinérgicamente en la refundición de ambos. A continuación, se presentan algunos ejemplos de actividades realizadas con el Método Cooperativo por Proyectos, que se ha llevado a la práctica en diferentes ámbitos educativos.

\footnotetext{
${ }^{5}$ Extraído el 16-10-16 de http://es.slideshare.net/norman.trujillo/el-mtodo-de-proyectos-como-tcnicadidctica
} 


\subsection{Aplicación en el ámbito universitario (Magisterio)}

Según la propia experiencia y la investigación-acción que se ha realizado en un contexto real, la idoneidad del método se contrastó, formando dos grupos de alumnos distintos a los que se impartieron los mismos contenidos, por una parte, a través de la mencionada metodología y por otra, mediante la Clase Magistral. La experiencia se estructuró sobre alumnos que ya tenían algunas nociones musicales a los cuales se les pasó un pretest para valorarlas. Cuando finalizó la actividad, se pasó un postest para estimar el nivel de aprendizaje adquirido tras la intervención. No obstante, y dado que el test es un elemento cuantificable excesivamente objetivo, hubo que añadir al mismo una encuesta de percepción a fin de que los valores como la cooperación, la implicación en el proyecto y otros elementos más subjetivos tuvieran cabida en su valoración final. El proyecto se llevó a cabo con la utilización de hidrófonos construidos por ellos mismos. Este proceso fue altamente educativo y potenciador de diversas habilidades y competencias transversales. La conclusión a la que se ha llegado es que en ambos casos los resultados fueron óptimos, si bien el Método por Proyectos resultó elegido por el alumnado por incluir una serie de elementos que lo hizo más estimulante, atractivo e incluso más productivo a largo plazo. Esto se pudo constatar cuando la experiencia finalizó mediante una evaluación cuantitativa y cualitativa respecto a la propuesta de experimentar con el Método Cooperativo por Proyectos. Como colofón de la experiencia se dieron las oportunas aplicaciones didácticas en la Educación Infantil y en la Educación Primaria.

\subsection{Aplicaciones en enseñanza secundaria obligatoria (ESO)}

La aplicación en ESO se ha producido a lo largo de diversos cursos, posibilitando la puesta en marcha de diferentes proyectos para cada uno de ellos. En estas edades suele ser muy llamativa la utilización de instrumentos no convencionales para hacer música y por ello, se han desarrollado actividades en dicha línea. No olvidemos que puede ser un fin interesante, estimular la escucha hacia sonidos poco habituales que despierten su atención y les sensibilicen hacia otros medios desconocidos.

Una de estas experiencias consistió en interpretar la obra Balloonology de Jeremi Telfort con globos. Esto permitió obtener sonidos que nunca hubieran sido elegidos como "música". La textura de la superficie hinchada puede restregarse, golpearse, hacerla chirriar... generando una gama de diferentes texturas sonoras muy interesantes a la par que originales.

En otra experiencia se utilizaron abanicos como instrumentos musicales. En este caso se hizo sobre la obra de Silvia León Aire rítmico. La textura del sonido fue más potente y espectacular que en el caso anterior, puesto que al golpearlos, frotarlos, cerrarlos suave o violentamente... crearon muchas más posibilidades sonoras y a los alumnos les pareció que avanzaban en un territorio inexplorado por ellos pero cuyo resultado artístico quedaba fuera de toda duda.

Otras propuestas experimentales se han llevado a cabo con el uso de materiales muy diversos como: periódicos, escobas, balones, mecheros... Lo cual pone de 
Botella Nicolás, A. M., y Adell Valero, J. R. Bases conceptuales para el diseño de una metodología de didáctica musical

manifiesto lo anteriormente expuesto. El trabajo Cooperativo por Proyectos, llevado a cabo con instrumentación no convencional, es una herramienta magnífica para trabajar el área de música en cualquier etapa educativa.

\section{REFERENCIAS}

Borrás Julibert, F. y Gómez Alemany, I. (2010). Dos experiencias de aprendizaje cooperativo: clase de instrumento y conjunto instrumental. Eufonía, 50, 109-120.

Botella, A. y Adell, R. (2016). Una propuesta experimental en la enseñanza de la música en secundaria. MAVAE: Cuadernos de Música, Artes Visuales y Artes Escénicas, 11, 67-71.

Clark, A. (2006). Changing Classroom Practice to Include the Project Aproach. Early Childhood Research \& Practice. ECRP, 8, 1-11.

Delalande, F. (1995). La música es un juego de niños. Buenos Aires: Ricordi Americana.

Echeita, G. (2012). El aprendizaje cooperativo al servicio de una educación de calidad. Cooperar para aprender y aprender a cooperar. En J. Torrego \& A. Negro (Eds.), Aprendizaje cooperativo en las aulas (pp. 21- 45). Madrid: Alianza editorial.

Hernández, F. y Ventura, M. (1998). La organización del curriculum y proyectos de trabajo. El conocimiento es un calidoscopio. Barcelona: Ice-Graó.

Ovejero, A. (1993). El aprendizaje cooperativo: Una eficaz aportación de la psicología social a la escuela del siglo XXI. Psicothema, 5, 373-391.

Pujolàs, P. (2012). La implantación del aprendizaje cooperativo en las aulas. En J. Torrego y A. Negro (Ed.), Aprendizaje cooperativo en las aulas (pp. 77- 104). Madrid: Alianza editorial.

Schafer, M. (2008). El rinoceronte en el aula. Buenos Aires: Melos.

Siegel, L. S. y Brainerd, C. J. (1978). Alternatives to Piaget: critical essays on the theory. New York: Academic Press.

Torrego, J. C. y Negro, A. (2012). Aprendizaje cooperativo en las aulas. Fundamentos y recursos para su implantación. Madrid: Alianza editorial.

\section{AUTORES:}

\section{Ana María Botella Nicolás:}

Doctora en pedagogía por la Universitat de València. Es Licenciada en Geografía e Historia, especialidad Musicología y maestra en Educación Musical, por la Universidad 
de Oviedo. Grado profesional en la especialidad de piano. Durante el año 2001 obtiene por oposición plaza en el cuerpo de profesores de música de enseñanza secundaria en Alicante (actualmente en excedencia). Ha presentado diversas comunicaciones en jornadas y congresos sobre didáctica de la música así como distintas publicaciones. Es profesora contratada doctora del departamento de didáctica de la Expresión Musical, Plástica y Corporal de la Facultad de Magisterio de la Universitat de València. Forma parte de la Comisión de Coordinación Académica del Master Universitario en Profesor/a de enseñanza secundaria de la UVEG y del Máster de Investigación en didácticas específicas. Desde Febrero de 2015 dirige el Aula de Música del Vicerrectorado de Cultura e Igualdad.

ana.maria.botella@uv.es

Orcid ID: https://orcid.org/0000-0001-5324-7152

ResearchID: N-1375-2014

\section{José Rafael Valero:}

Doctor en Música. Tesis: El aprendizaje cooperativo por proyectos en la educación musical universitaria: principios, planificación y exposición de experiencias. Licenciado con grado en Ciencias biológicas por la Universitat de València. Profesor superior de tuba, Conservatorio Joaquín Rodrigo de Valencia. Ejerce como profesor de Ciencias y de Música en ESO desde 1999. Actualmente es profesor de Florida Universitària (Universitat de València).

radell@florida-uni.es 